\title{
Scalar radius of the pion and $\gamma \gamma \rightarrow \pi \pi$
}

\section{Luis Roca*}

Departamento de Física. Universidad de Murcia. Spain, E-30071

E-mail: luisroca@um.es

\section{Jose Antonio Oller}

Departamento de Física. Universidad de Murcia. Spain, E-30071

E-mail: oller@um.es

\section{Carlos Schat}

Departamento de Física, FCEyN, Universidad de Buenos Aires. Ciudad Universitaria, Pab.1, (1428) Buenos Aires, Argentina.

E-mail: carlos-schat@gmail.com

\begin{abstract}
We make an improvement of the dispersion relation calculation of the quadratic scalar radius of the pion, $\left\langle r^{2}\right\rangle_{s}^{\pi}$, and the reaction $\gamma \gamma \rightarrow \pi^{0} \pi^{0}$. We solve a previous discrepancy between the the solution of the Muskhelishvili-Omnès equations for the non-strange null isospin $(I)$ pion scalar form factor and the Indurain's calculation using an Omnès representation of this form factor. We show that Ynduráin's method is indeed compatible with the determinations from the Muskhelishvili-Omnès equations once a possible zero in the scalar form factor is considered. Once this is accounted for, the resulting value is $\left\langle r^{2}\right\rangle_{s}^{\pi}=0.63 \pm 0.05 \mathrm{fm}^{2}$.

Regarding the reaction $\gamma \gamma \rightarrow \pi^{0} \pi^{0}$ we emphasize how the $f_{0}(980)$ signal emerges in $\gamma \gamma \rightarrow \pi \pi$ within the dispersive approach and how this fixes to a large extent the phase of the isoscalar Swave $\gamma \gamma \rightarrow \pi \pi$ amplitude above the $K \bar{K}$ threshold. This allows us to make sharper predictions for the cross section at lower energies and our results could then be used to distinguish between different $\pi \pi$ isoscalar S-wave parameterizations with the advent of new precise data on $\gamma \gamma \rightarrow$ $\pi^{0} \pi^{0}$. We also pay special attention to the role played by the $\sigma$ resonance in $\gamma \gamma \rightarrow \pi \pi$ and calculate its coupling and width to $\gamma \gamma$, for which we obtain $\Gamma(\sigma \rightarrow \gamma \gamma)=(1.68 \pm 0.15) \mathrm{KeV}$.
\end{abstract}

International Workshop on Effective Field Theories: from the pion to the upsilon February 2-6 2009

Valencia, Spain

\footnotetext{
*Speaker.
} 


\section{Introduction}

In the present contribution we summarize the three papers $[1,2,3]$ that mainly deal with the strong influence of the $I=0 \mathrm{~S}$-wave meson-meson final state interactions in the non-strange $I=0$ scalar form factor of the pion [1] and $\gamma \gamma \rightarrow \pi^{0} \pi^{0}$ [2]. Both processes can be formulated in a way that share in common the same basic function in order to take care of the strong final state interactions in the $I=0 \mathrm{~S}$-wave. This function has been recently the origin of large uncertainties in its implementation in the literature, both for the scalar form factor of the pion $[4,5,6]$ and for $\gamma \gamma \rightarrow \pi^{0} \pi^{0}[7]$.

Performing a Taylor expansion around $t=0$ of the scalar form factor of the pion, $\Gamma_{\pi}(t)$, $\Gamma_{\pi}(t)=\Gamma_{\pi}(0)\left\{1+\frac{1}{6} t\left\langle r^{2}\right\rangle_{s}^{\pi}+\mathscr{O}\left(t^{2}\right)\right\}$, the coeffcient of the linear term defines the quadratic scalar radius of the pion, $\left\langle r^{2}\right\rangle_{s}^{\pi}$. The quantity $\left\langle r^{2}\right\rangle_{s}^{\pi}$ contributes around $10 \%$ to the values of the S-wave $\pi \pi$ scattering lengths as determined in Ref. [8] by solving the Roy equations with constraints from two loop Chiral Perturbation Theory (CHPT). Related to that, $\left\langle r^{2}\right\rangle_{s}^{\pi}$ is also important in $S U(2) \times S U(2)$ CHPT since it gives the low energy constant $\bar{\ell}_{4}$ that controls the departure of $F_{\pi}$ from its value in the chiral limit $[9,10]$ at next-to-leading order. Based on one loop $\chi P T$, Gasser and Leutwyler [9] obtained $\left\langle r^{2}\right\rangle_{s}^{\pi}=0.55 \pm 0.15 \mathrm{fm}^{2}$. This calculation was improved later on by the same authors together with Donoghue [11], who solved the corresponding Muskhelishvili-Omnès equations with the coupled channels of $\pi \pi$ and $K \bar{K}$. The update of this calculation, performed in Ref. [8], gives $\left\langle r^{2}\right\rangle_{s}^{\pi}=0.61 \pm 0.04 \mathrm{fm}^{2}$. One should notice that solutions of the Muskhelishvili-Omnès equations for the scalar form factor consider only two coupled channels $(\pi \pi, K K)$ and have systematic uncertainties, specially for energies between $1-1.5 \mathrm{GeV}$ coming from the not consideration of the $4 \pi$, $6 \pi, 2 \eta$ and $\eta \eta^{\prime}$ states. Furthermore it relies on non-measured T-matrix elements or on assumptions about which are the channels that matter. Therefore, other independent approaches are then required. In this respect we quote the works [12, 13, 14], and Ynduráin's ones [4, 5, 6]. These latter works have challenged the previous value for $\left\langle r^{2}\right\rangle_{s}^{\pi}$, shifting it to the larger $\left\langle r^{2}\right\rangle_{s}^{\pi}=0.75 \pm 0.07 \mathrm{fm}^{2}$. If this is translated to the scattering lengths, it implies a shift of slightly more than one sigma. Refs. [4, 5] emphasize that one should have a precise knowledge of the $I=0$ S-wave phase shits, $\delta_{0}(s)$, for $s \geq 4 M_{K}^{2} \mathrm{GeV}^{2}, M_{K}$ is the kaon mass, to disentangle which of the values, either that of Ref. [8] or [4], is the right one. However, this point is based on an unstable behaviour of the solution of Ref. [4] with respect to the value of $\delta_{0}\left(4 M_{K}^{2}\right)$. Once this instability is cured, as shown below, the resulting $\left\langle r^{2}\right\rangle_{s}^{\pi}$ only depends weakly on $\delta_{0}(s), s \geq 4 M_{K}^{2}$, and is compatible with the value of Ref. [8].

Regarding the reaction $\gamma \gamma \rightarrow \pi^{0} \pi^{0}$ one has to emphasize that due to the absence of the Born term (since the $\pi^{0}$ is neutral), this reaction is specially sensitive to final state interactions. For energies below $0.6 \mathrm{GeV}$ or so, only the S-waves matter, which have $I=0$ or 2 . It is in this point where both the study of this reaction and the scalar form factor match. Recently, Ref. [7] updated the dispersive approach of Ref. [15] to calculate $\sigma\left(\gamma \gamma \rightarrow \pi^{0} \pi^{0}\right)$. Here one finds a large uncertainty in the results for $\sqrt{s} \geq 0.5 \mathrm{GeV}$ that at around $0.6 \mathrm{GeV}$ is already almost $200 \%$. This is due to the lack of a precise knowledge of the phase of the $\gamma \gamma \rightarrow \pi \pi I=0$ S-wave amplitude above $4 m_{K}^{2}$. We showed in Refs. [2,3] that one can largely remove the sensitivity for lower energies, $\sqrt{s} \lesssim 0.8 \mathrm{GeV}$, on the uncertainty in the not precisely known phase of the $I=0$ S-wave $\gamma \gamma \rightarrow \pi \pi$ amplitude above the $K \bar{K}$ threshold. The novelty was to include a further subtraction in the dispersion relation for the 
$I=0 \mathrm{~S}$-wave $\gamma \gamma \rightarrow \pi \pi$, together with an extra constraint to fix the additional subtraction constant. This was motivated by the use of an improved $I=0$ S-wave Omnès function. In Ref. [2,3] it is discussed in detail how the $f_{0}(980)$ peak, clearly seen recently in $\gamma \gamma \rightarrow \pi^{+} \pi^{-}$[16], can be generated within the dispersive method. As a result, the remaining ambiguity in this phase by $\pi$ in ref.[2] is removed and this allows to sharpen the prediction of the $\gamma \gamma \rightarrow \pi^{0} \pi^{0}$ cross section for $\sqrt{s} \lesssim 0.8 \mathrm{GeV}$, up to the onset of D-waves, as compared with ref.[2]. This could then be used to constraint further different parameterizations of the low energy $\pi \pi I=0 \mathrm{~S}$-wave.

\section{The scalar form factor of the pion}

Ref.[4] makes use of an Omnès representation for the pion scalar form factor,

$$
\Gamma_{\pi}(t)=P(t) \exp \left[\frac{t}{\pi} \int_{4 M_{\pi}^{2}}^{\infty} d s^{\prime} \frac{\phi_{0}\left(s^{\prime}\right)}{s^{\prime}\left(s^{\prime}-t-i \varepsilon\right)}\right] .
$$

Here, $P(t)$ is a polynomial in $t$ normalized such that $P(0)=\Gamma_{\pi}(0)$ and whose zeroes are those of $\Gamma_{\pi}(t)$. On the other hand, $\phi_{0}(t)$ is the continuous phase of $\Gamma_{\pi}(t) / P(t)$. Refs. [4, 5] make an assumption that is not always necessarily fulfilled. Namely, to identify $\phi_{0}(t)$ with the phase of $\Gamma_{\pi}(t)$, that we denote in the following as $\rho(t)$. If this identification is done it follows that $P(t)$ must be a constant. One must be aware that in Eq. (2.1) $\phi_{0}(t)$ is the phase of $\Gamma_{\pi}(t) / P(t)$. Notice that the phase of $\Gamma_{\pi}(t)$ is not continuous when crossing a zero located at $t_{1} \in \mathbb{R}$, since there is a flip in the sign when passing through. However, the phase of $\Gamma_{\pi}(t) / P(t)$ is continuous, since the zero is removed. This is the phase one should use in the Omnès representation, Eq. (2.1), because it results from a dispersion relation of $\log \Gamma_{\pi}(t) / P(t)$, and then $\phi(t)$ must be continuous (but not necessarily $\rho(t))$.

As stated, Ref. [4] took $\Gamma_{\pi}(t)=\Gamma_{\pi}(0) \exp \left[\frac{t}{\pi} \int_{4 M_{\pi}^{2}}^{\infty} d s^{\prime} \frac{\rho\left(s^{\prime}\right)}{s^{\prime}\left(s^{\prime}-t-i \varepsilon\right)}\right]$. So that the scalar form factor is given by, $\left\langle r^{2}\right\rangle_{s}^{\pi}=\frac{6}{\pi} \int_{4 M_{\pi}^{2}}^{+\infty} \frac{\rho(s)}{s^{2}} d s$. The phase $\rho(s)$ is fixed in Refs. [4, 5] by invoking Watson's final state theorem. For $s<s_{K}, s_{K}=4 M_{K}^{2}$, it implies that $\rho(s)=\delta_{0}(s)$, where neglecting inelasticity due to multipion states, an experimental fact. For $1.42>\sqrt{s} \gtrsim 1.1 \mathrm{GeV}$, Ref. [4] stressed the interesting fact that experimentally the inelasticity turns out to be small and hence Watson's final state theorem can be applied approximately again. In the narrow region between $2 M_{K}$ and $1.1 \mathrm{GeV}$ inelasticity cannot be neglected but Ref. [4] argues that, as it is so narrow, its contribution to Eq. (2) is small anyhow and, furthermore, that the elasticity parameter $\eta$ is not so small, so that one could still apply Watson's final state theorem with corrections. Finally, for $s>s_{0}=2 \mathrm{GeV}^{2}$ Ref. [4] takes a linear extrapolation from $\delta_{0}\left(s_{0}\right)$ to $\pi$. One should here criticize that it is still a long way to run from values of $\delta_{0}\left(s_{0}\right) \lesssim 2 \pi$ up to $\pi$ at $s \rightarrow+\infty$. With all these ingredients, and some error estimates, the value $\left\langle r^{2}\right\rangle_{s}^{\pi}=0.75 \pm 0.07 \mathrm{fm}^{2}$ results $[4,5]$.

The steps performed in Ref. [4] are not always compatible. In Ref. [1] we took as granted the assumption that Watson's final state theorem can be approximately applied for $1.5 \mathrm{GeV}>\sqrt{s}>$ $2 M_{K}$. Our assumption is in agreement with any explicit calculation of the pion non-strange $I=0$ scalar form factor. Now, Watson's final state theorem implies that $\phi(s)=\varphi(s)$ (modulo $\pi$ ), with $\varphi(s)$ the phase of the $I=0$ S-wave $\pi \pi$ amplitude, $t_{\pi \pi}=\left(\eta e^{2 i \delta_{0}}-1\right) / 2 i$. It occurs, as stressed in Refs. [17, 5], that $\varphi(s)$ can be either $\sim \delta_{0}(s)$ or $\sim \delta_{0}(s)-\pi$ depending on whether $\delta_{0}\left(s_{K}\right)>\pi$ or $<\pi$, respectively, for $s_{K}<s<2 \mathrm{GeV}^{2}$. The latter case corresponds to the calculation in Ref. [8], 
while the former is the preferred one in Ref. [5] and arguments are put forward for this preference in this reference. Let us evolve continuously from one situation $\left(\delta_{0}\left(s_{K}\right)<\pi\right)$ to the other $\left(\delta_{0}\left(s_{K}\right)>\pi\right)$. In the first case $\varphi(s)$ has an abrupt drop for $s>s_{K}$ simply because then $\eta<1$ and while the real part of $t_{\pi \pi}$ rapidly changes sign, its imaginary part is positive $(>0)$. The rapid movement in the real part is due to the swift one in $\delta_{0}(s)$ in the $K \bar{K}$ threshold due to the $f_{0}(980)$ resonance. As a result for $s \lesssim s_{K}, \varphi(s)=\delta_{0}(s) \simeq \pi$ and for $s \gtrsim s_{K}$ then $\varphi(s)<\pi / 2$. This rapid movement gives rise to a rapid drop in the Omnés function, Eq. (2), so that the modulus of the form factor has a deep minimum around $s_{K}$. Here, one is using Watson's final state theorem with $\phi_{0}(s)=\varphi(s)$ and the form factor of Ref. [11] is reproduced. Notice as well that in this case the function $\phi(s)$ approaches $\pi$ from below for asymptotic $s$ and then $P(t)=\Gamma_{0}(0)$ in Eq. (2.1). Now, we consider the limit $\delta_{0}(s) \rightarrow \pi^{-}$for $s \rightarrow s_{K}^{-}$. The superscript $-(+)$indicates that the limit is approached from below(above). In the limit, the change in sign in the real part of $t_{\pi \pi}$ occurs precisely at $s_{K}$, so that for $s=s_{K}^{-}, \varphi(s)=\pi$ and for $s=s_{K}^{+}$then $\varphi(s)<\pi / 2$. As a result one has a drop by $-\pi$ in $\varphi(s)$ which gives rise to a zero in the Omnès representation of the scalar form factor. Thus, the deep has evolved to a zero when $\delta_{0}\left(s_{K}\right) \rightarrow \pi^{-}$. Because of this zero the proper Omnès representation now involves a $P(t)=\Gamma_{\pi}(0)\left(1-t / s_{K}\right)$ and $\phi(s)$ is no longer $\varphi(s)$ but $\simeq \varphi(s)+\pi \simeq \delta_{0}(s)$ for $2.25 \mathrm{GeV}^{2}>s>s_{K}$. This follows simply because $\phi(s)$ is continuous. Thus, we go into a new realm where $\phi(s) \simeq \delta_{0}(s)$ and the degree of $P(t)$ is 1 , so that $\Gamma_{\pi}(t)$ has a zero at the point $s_{1}$ where $\delta_{0}\left(s_{1}\right)=\pi$ and $s_{1}<s_{K}$. Note that only at $s_{1}$ the imaginary part of $\Gamma_{\pi}(t)$ is zero and this fixes the position of the zero [1].

Hence for $\delta_{0}\left(s_{K}\right) \geq \pi$ one has to use

$$
\Gamma_{\pi}(t)=\Gamma_{\pi}(0)\left(1-\frac{t}{s_{K}}\right) \exp \left[\frac{t}{\pi} \int_{4 M_{\pi}^{2}}^{\infty} d s^{\prime} \frac{\phi\left(s^{\prime}\right)}{s^{\prime}\left(s^{\prime}-t-i \varepsilon\right)}\right]
$$

with $\phi(s) \simeq \delta_{0}(s)$ for $s<2.25 \mathrm{GeV}^{2}$. The uncertainties in this approximation for $s>s_{K}$ are discussed in Ref. [1] and included in the final error in $\left\langle r^{2}\right\rangle_{s}^{\pi}$.

Our final result is

$$
\left\langle r^{2}\right\rangle_{s}^{\pi}=0.63 \pm 0.05 \mathrm{fm}^{2} .
$$

The error takes into account different $\pi \pi I=0$ S-wave parameterizations, namely those of Refs. [8] and [18], the error in the application of Watson's final state theorem above $1 \mathrm{GeV}$ and up to $1.5 \mathrm{GeV}$, and the uncertainties in $\phi(s)$ given by asymptotic QCD for $s>2.25 \mathrm{GeV}^{2}$. This value is compatible with that of Ref. [8], $\left\langle r^{2}\right\rangle_{s}^{\pi}=0.61 \pm 0.04 \mathrm{fm}^{2}$, and also with $\left\langle r^{2}\right\rangle_{s}^{\pi}=0.64 \pm 0.06 \mathrm{fm}^{2}$ of Ref. [13] calculated from Unitary CHPT.

\section{3. $\gamma \gamma \rightarrow \pi \pi$}

In this section we report on the results of refs. [2, 3], where a more detailed account can be found. Let us consider the S-wave amplitude $\gamma \gamma \rightarrow(\pi \pi)_{I}, F_{I}(s)$, where the two pions have definite $I=0$ or 2 . The function $F_{I}(s)$ on the complex $s-$ plane is analytic except for two cuts along the real $s$-axis, the unitarity one for $s \geq 4 m_{\pi}^{2}$ and the left hand cut for $s \leq 0$, with $m_{\pi}$ the pion mass. Let us denote by $L_{I}(s)$ the complete left hand cut contribution to $F_{I}(s)$. Then, the function $F_{I}(s)-L_{I}(s)$, by construction, has only right hand cut. Let $\phi_{I}(s)$ be the phase of $F_{I}(s)$ modulo $\pi$, chosen in 
such a way that $\phi_{I}(s)$ is continuous and $\phi_{I}\left(4 m_{\pi}^{2}\right)=0$. For the exotic $I=2 \mathrm{~S}$-wave one can invoke Watson's final state theorem ${ }^{\# 1}$ so that $\phi_{2}(s)=\delta_{\pi}(s)_{2}$. For $I=0$ the same theorem guarantees that $\phi_{0}(s)=\delta_{\pi}(s)_{0}$ for $s \leq 4 m_{K}^{2}$, where we denote by $\delta_{\pi}(s)_{I}$ the isospin $I S$-wave $\pi \pi$ phase shifts. Here one neglects the inelasticity due to the $4 \pi$ and $6 \pi$ states below the two kaon threshold. Above the two kaon threshold $s_{K}=4 m_{K}^{2}$, the phase function $\phi_{0}(s)$ cannot be fixed a priori due the onset of inelasticity. However, as remarked in refs.[4, 1], inelasticity is again small for $\sqrt{s} \gtrsim 1.1 \mathrm{GeV}$, and one can then apply approximately Watson's final state theorem which implies that $\phi_{0}(s) \simeq \delta^{(+)}(s)$ modulo $\pi$. Here $\delta^{(+)}(s)$ is the eigenphase of the $\pi \pi, K \bar{K} I=0 \mathrm{~S}$-wave S-matrix such that it is continuous and $\delta^{(+)}\left(s_{K}\right)=\delta_{\pi}\left(s_{K}\right)_{0}$. In refs.[5, 1] it is shown that $\delta^{(+)}(s) \simeq \delta_{\pi}(s)_{0}$ or $\delta_{\pi}(s)_{0}-\pi$, depending on whether $\delta_{\pi}\left(s_{K}\right)_{0} \geq \pi$ or $<\pi$, respectively. In order to fix the integer factor in front of $\pi$ in the relation $\phi_{0}(s) \simeq \delta^{(+)}(s)$ modulo $\pi$, it is necessary to follow the possible trajectories of $\phi_{0}(s)$ in the narrow region $1 \lesssim \sqrt{s} \lesssim 1.1 \mathrm{GeV}$. The remarkable physical effects happening there are the appearance of the $f_{0}(980)$ resonance on top of the $K \bar{K}$ threshold and the cusp effect of the latter that induces a discontinuity at $s_{K}$ in the derivative of observables. Between 1.05 to $1.1 \mathrm{GeV}$ there are no further narrow structures and observables evolve smoothly. Approximately half of the region between 0.95 and $1.05 \mathrm{GeV}$ is elastic and $\phi_{0}(s)=\delta_{\pi}(s)_{0}$ (Watson's theorem), so that it raises rapidly. Above $2 m_{K} \simeq 1 \mathrm{GeV}$ and up to $1.05 \mathrm{GeV}$ the function $\phi_{0}(s)$ can keep increasing with energy, like $\delta_{\pi}(s)_{0}$. The other possibility is a change of sign in the slope at $s_{K}$ due to the $K \bar{K}$ cusp effect such that $\phi_{0}(s)$ starts a rapid decrease in energy. Above $\sqrt{s}=1.05 \mathrm{GeV}, \phi_{0}(s)$ matches smoothly with the behaviour for $\sqrt{s} \gtrsim 1.1 \mathrm{GeV}$, which is constraint by Watson's final state theorem. As a result, for $\sqrt{s} \gtrsim 1 \mathrm{GeV}$ either $\phi_{0}(s) \simeq \delta_{\pi}(s)_{0}$ or $\phi_{0}(s) \simeq \delta_{\pi}(s)-\pi$, corresponding to an increasing or decreasing $\phi_{0}(s)$ above $s_{K}$, in order.

Let us define the switch $z$ to characterize the behaviour of $\phi_{0}(s)$ for $s>s_{K}$, and close to $s_{K}$, such that $z=+1$ if $\phi_{0}(s)$ rises with energy and $z=-1$ if it decreases. Let $s_{1}$ be the value of $s$ at which $\phi_{0}\left(s_{1}\right)=\pi$. Following ref.[1] we introduce the Omnès function,

$$
\Omega_{0}(s)=\left(1-\theta(z) \frac{s}{s_{1}}\right) \exp \left[\frac{s}{\pi} \int_{4 m_{\pi}^{2}}^{\infty} \frac{\phi_{0}\left(s^{\prime}\right)}{s^{\prime}\left(s^{\prime}-s\right)} d s^{\prime}\right],
$$

with $\theta(z)=1$ for $z=+1$ and 0 for $z=-1$. Given the definition of the phase function $\phi_{I}(s)$ the function $F_{I}(s) / \Omega_{I}(s)$ has no right hand cut. Next, we perform a twice subtracted dispersion relation for $\left(F_{0}(s)-L_{0}(s)\right) / \Omega_{0}(s)$

$F_{0}(s)=L_{0}(s)+c_{0} s \Omega_{0}(s)+\frac{s^{2}}{\pi} \Omega_{0}(s) \int_{4 m_{\pi}^{2}}^{\infty} \frac{L_{0}\left(s^{\prime}\right) \sin \bar{\phi}_{0}\left(s^{\prime}\right)}{s^{\prime 2}\left(s^{\prime}-s\right)\left|\Omega_{0}\left(s^{\prime}\right)\right|} d s^{\prime}+\theta(z) \frac{\omega_{0}(s)}{\omega_{0}\left(s_{1}\right)} \frac{s^{2}}{s_{1}^{2}}\left(F_{0}\left(s_{1}\right)-L_{0}\left(s_{1}\right)\right)$,

where $\omega_{0}(s)=\exp \left[\frac{s}{\pi} \int_{4 m_{\pi}^{2}}^{\infty} \frac{\phi_{0}\left(s^{\prime}\right)}{s^{\prime}\left(s^{\prime}-s\right)} d s^{\prime}\right]$. In the previous equation we introduce $\bar{\phi}_{0}(s)$ that is defined as the phase of $\Omega_{0}(s)$. Proceeding similarly for $I=2$ one has

$$
F_{2}(s)=L_{2}(s)+c_{I} s \Omega_{2}(s)+\frac{s^{2}}{\pi} \Omega_{2}(s) \int_{4 m_{\pi}^{2}}^{\infty} \frac{L_{2}\left(s^{\prime}\right) \sin \phi_{2}\left(s^{\prime}\right)}{s^{\prime 2}\left(s^{\prime}-s\right)\left|\Omega_{2}\left(s^{\prime}\right)\right|} d s^{\prime} .
$$

It is worth mentioning that eq.(3.2) for $I=0$ and $z=+1$ is equivalent to perform a three times subtracted dispersion relation for $\left(F_{0}(s)-L_{0}(s)\right) / \omega_{0}(s)$. Let us denote by $F_{N}(s)$ the S-wave

\footnotetext{
${ }^{\# 1}$ This theorem implies that the phase of $F_{I}(s)$ when there is no inelasticity is the same, modulo $\pi$, as the one of the isospin $I \mathrm{~S}$-wave $\pi \pi$ elastic strong amplitude.
} 


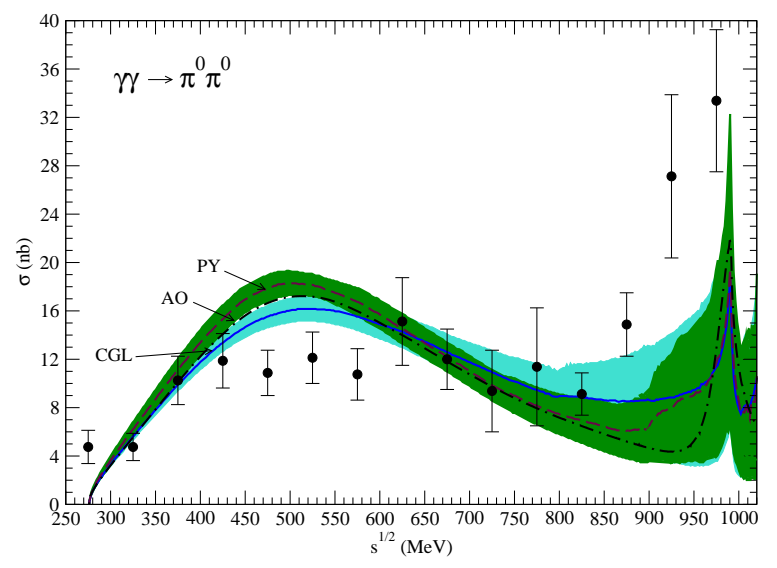

Figure 1: Final result for the $\gamma \gamma \rightarrow \pi^{0} \pi^{0}$ cross for $\sqrt{s} \leq 1.05 \mathrm{GeV}$. The experimental data are from the Crystal Ball Collaboration [20].

$\gamma \gamma \rightarrow \pi^{0} \pi^{0}$ amplitude and by $F_{C}(s)$ the $\gamma \gamma \rightarrow \pi^{+} \pi^{-}$one. We are still left with the unknown subtraction constants $c_{0}, c_{2}$ for $I=0$ and 2 , respectively, and $F_{0}\left(s_{1}\right)-L_{0}\left(s_{1}\right)$ for $I=0$ and $z=+1$. The $c_{0}, c_{2}$, constants can be obtained from low energy theorems and matching to one loop $\chi \mathrm{PT}$. The value of $F_{0}\left(s_{1}\right)-L_{0}\left(s_{1}\right)$ can be restricted because the cross section $\sigma\left(\gamma \gamma \rightarrow \pi^{0} \pi^{0}\right)$ around the $f_{0}(980)$ resonance is quite sensitive to this constant. We impose that $\sigma\left(\gamma \gamma \rightarrow \pi^{0} \pi^{0}\right) \leq 40 \mathrm{nb}$ at $s_{1}$. This upper bound for the peak of the $f_{0}(980)$ in $\gamma \gamma \rightarrow \pi^{0} \pi^{0}$ is equivalent to impose that the $\gamma \gamma$ width of the $f_{0}(980)$ lies in the range $205_{-83}^{+95}(\text { stat })_{-117}^{+147}($ sys $) \mathrm{eV}$ as determined in ref.[16]. We shall see that the effect of this rather large uncertainty allowed at $1 \mathrm{GeV}$, see fig.1, is very mild at lower energies. As the $f_{0}(980)$ resonance gives rise to a small peak in the precise data on $\gamma \gamma \rightarrow \pi^{+} \pi^{-}$ [16], then $\phi_{0}(s)$ must increase with energy above $s_{K}$ and the case with $z=+1$ is the one realized in nature. Note that for $z=-1$ in eq.(3.2), there is no a local maximum associated with this resonance in $\left|F_{0}(s)\right|$ but a minimum, because $\left|\omega_{0}(s)\right|$ has a dip around the $f_{0}(980)$ mass.

The source of uncertainty in the approximate relation $\phi_{0}(s) \simeq \delta_{\pi}(s)_{0}$ for $4 m_{K}^{2} \lesssim \sqrt{s} \lesssim 1.5 \mathrm{GeV}$ and its functional dependence for $s>s_{H}=2.25 \mathrm{GeV}^{2}$ is estimated similarly as in ref.[3, 1]. In fig.1 we show our final results for the $\gamma \gamma \rightarrow \pi^{0} \pi^{0}$, where the band around each line corresponds to the estimated error. The error band for the dot-dashed line is not shown because it is similar to the ones of the other two curves. In this figure PY refers to using the $I=0 \mathrm{~S}$-wave $\pi \pi$ of ref.[18], CGL that of ref.[8] and AO the one of ref.[19]. One observes that for $\sqrt{s} \lesssim 0.8 \mathrm{GeV}$ the uncertainty in the loose bound for the $f_{0}(980)$ greatly disappears. For such energies the main source of uncertainty originates from the uncertainties in the $\pi \pi$ phase parameterizations used.

The previous model allows dor an evaluation of the $\sigma \rightarrow \gamma \gamma$ width. The coupling $\sigma \rightarrow \gamma \gamma$, $g_{\sigma \gamma \gamma}$, can be evaluated from the residue of the amplitude $F_{N}(s)$ at the second Riemann sheet. It can be easily obtained that

$$
\frac{g_{\sigma \gamma \gamma}^{2}}{g_{\sigma \pi \pi}^{2}}=-\frac{1}{2}\left(\frac{\sigma_{\pi}\left(s_{\sigma}\right)}{8 \pi}\right)^{2} F_{0}\left(s_{\sigma}\right)^{2}
$$

where $g_{\sigma \pi \pi}$ is the coupling of the $\sigma$ to $\pi \pi$.

We denote by $s_{\sigma}=\left(M_{\sigma}-i \Gamma_{\sigma} / 2\right)^{2}$. Ref.[21] provides $M_{\sigma}^{C C L}=441_{-8}^{+16} \mathrm{MeV}$ and $\Gamma_{\sigma}^{C C L}=$ $544_{-25}^{+18} \mathrm{MeV}$, while from ref.[19] one has $M_{\sigma}^{A O}=(456 \pm 6) \mathrm{MeV}$ and $\Gamma_{\sigma}^{A O}=(482 \pm 20) \mathrm{MeV}$. In 
the following the superscripts $A O$ and $C C L$ refer to those results obtained by employing $s_{\sigma}$ from ref.[19] or [21], respectively. From eq.(3.4) we obtain $\left|g_{\sigma \gamma \gamma} / g_{\sigma \pi \pi}\right|=2.01 \pm 0.11$ for $s_{\sigma}^{C C L}$ and $1.85 \pm 0.09$ for $s_{\sigma}^{A O}$. Given $s_{\sigma}$, this ratio of residua is the well defined prediction that follow from our $F_{0}(s)$. We employ the standard narrow resonance width formula in terms of $g_{\sigma \gamma \gamma}$ to calculate $\Gamma(\sigma \rightarrow \gamma \gamma)=\frac{\left|g_{\sigma \gamma \gamma}\right|^{2}}{16 \pi M_{\sigma}}$. One needs to provide numbers for $\left|g_{\sigma \pi \pi}\right|$ in order to apply the previous equation and the determined $\left|g_{\sigma \gamma \gamma} / g_{\sigma \pi \pi}\right|$. We first consider the value $\left|g_{\sigma \pi \pi}^{A O}\right|=(3.17 \pm 0.10) \mathrm{GeV}$ from the approach of ref.[19]. The calculated width is $\Gamma^{A O}(\sigma \rightarrow \pi \pi)=(1.50 \pm 0.18) \mathrm{KeV}$. Not only the position of the pole in the partial wave amplitude, but also its residue can be calculated in the framework of the dispersive analysis described in ref.[21]. Expressed in terms of the complex coefficient $g_{\sigma \pi \pi}$, the preliminary result for the residue amounts to $\left|g_{\sigma \pi \pi}^{C C L}\right|=\left(3.31_{-0.08}^{+0.17}\right) \mathrm{GeV}$, $\Gamma^{C C L}(\sigma \rightarrow \gamma \gamma)=\left(1.98_{-0.24}^{+0.30}\right) \mathrm{KeV}$. Taking the average between these two values for $\Gamma(\sigma \rightarrow \gamma \gamma)$ we end with,

$$
\Gamma(\sigma \rightarrow \gamma \gamma)=(1.68 \pm 0.15) \mathrm{KeV}
$$

\section{References}

[1] J. A. Oller and L. Roca, Phys. Lett. B651, 139 (2007).

[2] J. A. Oller, L. Roca and C. Schat, Phys. Lett. B 659 (2008) 201 [arXiv:0708.1659 [hep-ph]].

[3] J. A. Oller and L. Roca, Eur. Phys. J. A 37 (2008) 15 [arXiv:0804.0309 [hep-ph]].

[4] F. J. Ynduráin, Phys. Lett. B578, 99 (2004); (E)-ibid B586, 439 (2004).

[5] F. J. Ynduráin, Phys. Lett. B612, 245 (2005).

[6] F. J. Ynduráin, arXiv:hep-ph/0510317.

[7] M. R. Pennington, Phys. Rev. Lett. 97, 011601 (2006).

[8] G. Colangelo, J. Gasser and H. Leutwyler, Nucl. Phys. B603, 125 (2001).

[9] J. Gasser and H. Leutwyler, Phys. Lett. B125, 325 (1983).

[10] G. Colangelo and S. Dür, Eur. Phys. J. C33, 543 (2004).

[11] J. F. Donoghue, J. Gasser and H. Leutwyler, Nucl. Phys. B343, 341 (1990).

[12] J. Gasser and U.-G. Meißner, Nucl. Phys. B357, 90 (1991).

[13] U. G. Meißner and J. A. Oller, Nucl. Phys. A679, 671 (2001).

[14] J. Bijnens, G. Colangelo and P. Talavera, JHEP 9805, 014 (1998).

[15] D. Morgan and M. R. Pennington, Phys. Lett. B272, 134 (1991); Z. Phys. C37, 431 (1988) [Erratum-ibid. C 39, 590 (1988)].

[16] T. Mori et al. [Belle Collaboration], Phys. Rev. D75 (2007) 051101.

[17] B. Ananthanarayan, I. Caprini, G. Colangelo, J. Gasser and H. Leutwyler, Phys. Lett. B602, 218 (2004).

[18] J. R. Peláez and F. J. Ynduráin, Phys. Rev. D68, 074005 (2003); ibid D71, 074016 (2005).

[19] M. Albaladejo and J. A. Oller, Phys. Rev. Lett. 101 (2008) 252002 [arXiv:0801.4929 [hep-ph]].

[20] H. Marsiske et al. [Crystal Ball Collab.], Phys. Rev. D41, 3324 (1990).

[21] I. Caprini, G. Colangelo and H. Leutwyler, Phys. Rev. Lett. 96 (2006) 132001. 